\title{
Severity of cardiovascular disease and health-related quality of life in men with prostate cancer: a longitudinal analysis from CaPSURE
}

\author{
Lonneke V. van de Poll-Franse · Natalia Sadetsky • \\ Lorna Kwan · Mark S. Litwin
}

Accepted: 28 April 2008/Published online: 28 May 2008

(C) The Author(s) 2008

\begin{abstract}
Objective To evaluate the influence of comorbid cardiovascular disease severity on health-related quality of life (HRQL) in men treated with radical prostatectomy (RP) or radiotherapy (RT) for early stage prostate cancer.

Methods Subjects $(n=830)$ with non-metastatic disease who had been diagnosed in 2000-2002 were drawn from Cancer of the Prostate Strategic Urologic Research Endeavor (CaPSURE). We evaluated the influence of cardiovascular disease (CVD) severity on generic and disease-specific HRQL before and 6, 12, 18, and 24 months after treatment with RP or RT. HRQL was measured with the SF-36 and the UCLA Prostate Cancer Index.
\end{abstract}

L. V. van de Poll-Franse $(\bowtie)$

Comprehensive Cancer Centre South (IKZ), Eindhoven Cancer Registry, P.O. Box 231, 5600 AE Eindhoven, The Netherlands e-mail: 1.vd.poll@ikz.nl

L. V. van de Poll-Franse

Center of Research on Psychology in Somatic Diseases

(CoRPS), Tilburg University, Tilburg, The Netherlands

L. V. van de Poll-Franse · L. Kwan - M. S. Litwin Jonsson Comprehensive Cancer Center, University of California, Los Angeles, Los Angeles, CA, USA

N. Sadetsky

Department of Urology, University of California, San Francisco, San Francisco, CA, USA

M. S. Litwin

Department of Urology, David Geffen School of Medicine at UCLA, University of California, Los Angeles, Los Angeles, CA, USA

M. S. Litwin

Department of Health Services, School of Public Health,

University of California, Los Angeles, Los Angeles, CA, USA
Results Men with moderate $(n=193)$ or severe $(n=51)$ cardiovascular disease had worse pre-treatment HRQL than did men without CVD $(n=293)(P<0.01)$; HRQL scores were worse in men referred for RT. During 24 months of follow-up, men with moderate or severe CVD had worse SF-36 physical and mental component summaries and worse bowel function at all time points $(P<0.05)$. Men with severe CVD also experienced a slower recovery in physical function $(P=0.03)$ and sexual functioning $(P=0.02)$ than did men without CVD.

Conclusions Prostate cancer patients with moderate to severe CVD have worse HRQL during follow-up. Those with severe CVD recover their physical and sexual functioning more slowly after treatment.

Keywords Prostate cancer - Cardiovascular disease . Health-related quality of life

Because most men are older at the time of diagnosis with prostate cancer [1], many have comorbid medical conditions. Hence, cancer treatment decisions are made in the context of an older individual's pre-existing comorbidity in order to provide high quality cancer care. In 2001 the National Institute on Aging (NIA) and the National Cancer Institute (NCI) recognized this and issued recommendations for studies on comorbidity and cancer in older patients [2]. Since then, comorbidity in prostate cancer has been studied in relation to treatment [3-6], early complications after radical prostatectomy [7], and overall survival [5-7], providing valuable new insights into the importance of comorbidity when considering treatment and prognosis in older prostate cancer patients. Unfortunately, most of these studies assessed comorbidity with a checklist as being present or absent, or incorporated a simple count of 
conditions, not taking into account the severity of disease. Also, these studies focused on major comorbid conditions, such as diabetes, heart disease or gastrointestinal problems, whereas more common problems, such as hypertension, that can affect daily activity ought to be included as well [2].

Because the majority of men with prostate cancer do not die of it [8], accurate assessment of comorbid conditions can inform treatment decisions. However, the impact of comorbidity on health-related quality of life (HRQL) in prostate cancer patients has rarely been reported [9-11] and is among the factors often not well-described in HRQL outcomes studies [12]. A previous CaPSURE analysis showed that men with heart disease showed a greater worsening in their physical functioning after radical prostatectomy [9], whereas a study among prostate cancer patients treated with radiotherapy revealed gastro-intestinal complications associated with the prevalence of diabetes [13]. Both studies suggest that there could be an interaction among co-morbidity, prostate cancer treatment, and HRQL.

Our objective was to evaluate the influence of comorbid cardiovascular disease on HRQL in men treated with radical prostatectomy or radiotherapy for early stage prostate cancer. Cardiovascular disease (CVD), the most prevalent comorbid condition in prostate cancer patients [5], ranges broadly in severity from controlled hypertension to myocardial infarction or congestive heart failure. To assess CVD severity, we used the validated Total Illness Burden Index for Prostate Cancer (TIBI-CaP) [14], a patientreported measure of comorbidity specifically developed for the assessment of severity of concurrent disease in prostate cancer patients. We evaluated the influence of CVD on generic and disease-specific HRQL before and 6, 12, 18, and 24 months after treatment for patients with no, mild, moderate, or severe CVD according to type of treatment. We hypothesized that increasing severity of CVD would be associated with worse baseline generic and disease-specific HRQL and slower recovery of HRQL during 24 months of follow-up.

\section{Methods}

\section{Patients}

The study sample was drawn from Cancer of the Prostate Strategic Urologic Research Endeavor (CaPSURE), a longitudinal, observational registry of men with prostate cancer. Data are collected from participant questionnaires and medical records at 34 community-based practices, 3 academic and 3 Veterans Administration clinical sites across the US. Clinical data (medical history, tumor stage, prostatespecific antigen [PSA], and treatment) are provided by the urologist or radiation oncologist at clinical encounters. Subjects report HRQL data at entry and semiannually until death or withdrawal from the study. Additional details of the project methods have been previously reported [15]. In addition to the semiannual questionnaires, at the end of 2002 the Total Illness Burden Index for Prostate Cancer (TIBI$\mathrm{CaP}$ ) was sent to 4,635 active CaPSURE participants, of whom 3,409 (74\%) returned the questionnaire [14].

For this analysis, we included all subjects diagnosed in 2000-2002 with non-metastatic disease (clinical stage T1-T3) and who underwent radical prostatectomy (RP) or radiation therapy (RT; external beam or brachytherapy). All subjects completed questionnaires pretreatment and at least twice within 24 months after treatment. All subjects completed the TIBI-CaP within the same 24 months after treatment. The final analysis included 830 men, of whom 78 had 2, 216 had 3, and 536 had 4 post-treatment questionnaires, yielding 3,778 questionnaires.

Measures

\section{Risk of prostate cancer recurrence}

Subjects were stratified according to pre-treatment risk using a modification of the D'Amico risk classification [16], namely high (PSA greater than $20 \mathrm{ng} / \mathrm{ml}$, Gleason sum greater than 7 , primary Gleason pattern 4 or 5 , or clinical stage T3a), intermediate (PSA 10.1-20 ng/ml, Gleason sum 7, secondary Gleason pattern 4 or 5 , or clinical stage $\mathrm{T} 2 \mathrm{~b} / \mathrm{c}$ ), or low (PSA $10 \mathrm{ng} / \mathrm{ml}$ or less, Gleason sum less than 7 without pattern 4 or 5 , and clinical stage $\mathrm{T} 1$ or $\mathrm{T} 2 \mathrm{a})$.

\section{Comorbidity}

The TIBI-CaP [14], a patient-reported measure of comorbidity, was used to measure the presence and severity of comorbid conditions. The developers of the TIBI-CaP modified the original TIBI [17] based on a pilot study [18] and then established its validity in 2,894 CaPSURE participants by showing that it accurately predicted both mortality and future quality of life [14]. The TIBI-CaP includes 84 items in 11 sub-dimensions: pulmonary disease and congestive heart failure, heart disease (heart attack, coronary artery bypass, coronary angioplasty), stroke and neurologic disease, gastrointestinal conditions, other cancers (excluding prostate), arthritis, foot and leg conditions, eye and vision conditions, hearing problems, hypertension, and diabetes. Using a scoring algorithm that assigns points based on severity of illness, scores are derived for each of the 11 sub-dimensions, with higher scores corresponding to greater severity of comorbidities. 
We combined three sub-domains-congestive heart failure (CHF), atherosclerotic heart disease (ASHD), and hypertension-into one cardiovascular disease severity score. In accordance with the TIBI-CaP scoring system, we weighted the sub-dimensions differentially. ASHD and $\mathrm{CHF}$, both considered to have the greatest clinical impact on illness burden, were stratified based on clinical judgment into four severity levels (0-3 points), whereas hypertension, considered to have the least impact, was stratified in two severity levels (no or controlled vs uncontrolled hypertension). The severity weights for each sub-dimension were summed to create a TIBI-CVD score, which could range from 0 to 7 . Scores in our sample ranged from 0 to 7 with a mean of 1.2 (standard deviation, 1.3) and a median of 1 . For example, an individual with angina or previous myocardial infarction without current complaints earned one point, but if he reported chest pain at rest, he earned three ASHD points. Or, a subject who reported shortness of breath at rest all the time in the presence of swollen ankles most of the day earned three CHF points. Based on clinical judgment and after comparing the baseline RAND 36 domains most closely related to chronic disease severity (physical functioning and role limitations - physical) as an indicator of general health status, the TIBI-CVD score was stratified as no CVD (0 points), mild CVD (1 point), moderate CVD (2-3 points), and severe CVD (4-7 points).

\section{Health-related quality of life}

We measured general HRQL with the RAND 36-Item Health Survey, version 1.0, a 36-item questionnaire that quantifies general physical and mental HRQOL [19]. In this analysis, we examined the domains of physical function, general health, bodily pain, vitality, social functioning, mental health, role-physical (limitations due to physical problems), role-emotional (limitations due to emotional problems), and the physical and mental component summary scores. Differences between subgroups of at least $0.5 \mathrm{SD}$ were considered clinically meaningful. We measured disease-specific HRQL with the UCLA Prostate Cancer Index (PCI) [20]. This self-administered, 20-item questionnaire quantifies prostate cancer-specific HRQL in six domains, including urinary, bowel, and sexual function and bother. We examined only function (urinary, sexual, and bowel function) and not bother. All PCI outcomes are scored so that higher values indicate better quality of life.

\section{Statistical analysis}

We compared baseline demographic and clinical variables across TIBI-CVD severity level with ANOVA for continuous variables and chi-square analyses for categorical variables. We carried out linear regression analyses to evaluate the association between baseline demographic and clinical characteristics and HRQL. Variables were included in the analysis of covariance (ANCOVA) if they showed a univariate association $(P<0.05)$ with HRQL outcomes.

From longitudinal studies, it is well known that most subjects report a decline in HRQL right after treatment and then experience a gradual improvement, resulting in a negative and positive regression slope when evaluating HRQL from baseline to 24 months. In order to understand the process of HRQL decline after treatment and then recovery after a while, we separately analyzed the decline of HRQL and then recovery. These separate analyses also resulted in better fitting models.

We used ANCOVA to compare mean baseline and change in HRQL scores within the first 6 months across levels of TIBI-CVD severity. Covariates were selected based on prior knowledge and previous CaPSURE analyses on comorbidity and HRQL $[9,11]$. In addition, we incorporated baseline HRQL scores as a covariate for quality of life in all models, as they are known to correlate strongly with outcomes over time [21, 22]. All of these variables appeared to be independently associated $(P<0.05)$ with HRQL.

We used repeated measures analyses with mixed modeling to examine changes in HRQL between 6 and 24 months after diagnosis. Repeated measures analysis takes into account the correlation of repeated outcomes among subjects and handles missing values in an optimal way by accounting for the time patterns of the available data. We evaluated the positive regression slope, assessing HRQL at 6, 12, 18, and 24 months, but incorporated baseline HRQL scores as a covariate for quality of life in all models, as they are known to correlate strongly with outcomes over time [21, 22]. Covariates were selected based on prior knowledge and previous CaPSURE analyses on comorbidity and HRQL [9, 11]. All of these variables appeared to be independently associated $(P<0.05)$ with HRQL. We also tested interaction terms for CVD severity and time and CVD severity and treatment to evaluate whether CVD severity had a different effect on HRQL over time or for different therapies.

As comorbidity has been associated with less aggressive treatment [3-6], and a previous CaPSURE analysis revealed that men with CVD were more likely to be treated with radiotherapy than prostatectomy, we separately analyzed baseline and follow-up HRQL for the two treatments.

We used $P<0.05$ to report statistical significance and made no formal adjustment for multiple testing. All statistical analyses were performed in SAS 9.1 (SAS Institute Inc., Cary, NC). 
Table 1 Demographic and medical characteristics for prostate cancer patients according to their TIBI-CaP cardiovascular disease severity score

\begin{tabular}{|c|c|c|c|c|c|}
\hline \multirow[t]{2}{*}{ Characteristic } & \multicolumn{5}{|c|}{ TIBI-CaP cardiovascular disease severity } \\
\hline & No $(n=293)$ & Mild $(n=293)$ & Moderate $(n=193)$ & Severe $(n=51)$ & $P$-value \\
\hline Age (mean years) & $63.7 \pm 7.8$ & $64.9 \pm 7.8$ & $66.9 \pm 7.3$ & $67.2 \pm 7.1$ & $<0.0001$ \\
\hline \multicolumn{6}{|l|}{ Ethnicity } \\
\hline White $(\%)$ & $273(93)$ & $276(94)$ & $187(97)$ & $45(88)$ & 0.10 \\
\hline \multicolumn{6}{|l|}{ Partner status ${ }^{\mathrm{a}}(\%)$} \\
\hline Partnered & $265(91)$ & 271 (93) & $175(92)$ & $43(84)$ & 0.24 \\
\hline \multicolumn{6}{|l|}{ Education level $^{\mathrm{b}}(\%)$} \\
\hline Less than high school & $108(38)$ & $95(33)$ & $79(42)$ & $25(51)$ & 0.06 \\
\hline High school graduate & $45(16)$ & $59(20)$ & $39(21)$ & $12(24)$ & \\
\hline Some college & $50(17)$ & $51(18)$ & $28(15)$ & $6(12)$ & \\
\hline College graduate & $84(29)$ & $84(29)$ & $41(22)$ & $6(12)$ & \\
\hline \multicolumn{6}{|l|}{$\operatorname{Income}^{\mathrm{c}}(\%)$} \\
\hline$<30,000$ & $68(27)$ & $58(21)$ & $51(29)$ & $17(34)$ & 0.06 \\
\hline $30-50,000$ & $65(25)$ & $72(27)$ & $47(27)$ & $17(34)$ & \\
\hline $50-70,000$ & $48(19)$ & $51(19)$ & $40(23)$ & $4(8)$ & \\
\hline$>75,000$ & $75(29)$ & 89 (33) & $35(20)$ & $12(24)$ & \\
\hline \multicolumn{6}{|l|}{ Insurance $(\%)$} \\
\hline Medicare supplement & $86(30)$ & $96(33)$ & $70(36)$ & $20(39)$ & 0.13 \\
\hline Medicare & $36(12)$ & $33(11)$ & $35(18)$ & $9(18)$ & \\
\hline Private insurance & $149(51)$ & $144(49)$ & $73(38)$ & $18(35)$ & \\
\hline Other/none & $22(8)$ & $20(7)$ & $15(8)$ & $4(8)$ & \\
\hline \multicolumn{6}{|l|}{ PSA (ng/ml) at diagnosis } \\
\hline Mean \pm SD & $8.0 \pm 8.3$ & $6.8 \pm 5.4$ & $8.2 \pm 8.2$ & $7.6 \pm 6.2$ & 0.10 \\
\hline Median & 6.0 & 5.5 & 6.2 & 5.6 & \\
\hline \multicolumn{6}{|l|}{ Biopsy Gleason score } \\
\hline Mean \pm SD & $6.3 \pm 0.7$ & $6.3 \pm 0.8$ & $6.5 \pm 0.8$ & $6.4 \pm 0.7$ & 0.16 \\
\hline Median & 6.0 & 6.0 & & & \\
\hline \multicolumn{6}{|l|}{ Tumor stage (\%) } \\
\hline 1 & $158(54)$ & $153(52)$ & $103(53)$ & $26(51)$ & 0.73 \\
\hline 2 & $129(44)$ & $138(47)$ & $88(46)$ & $25(49)$ & \\
\hline 3 & $6(2)$ & $2(1)$ & $2(1)$ & 0 & \\
\hline \multicolumn{6}{|c|}{ Recurrence risk at diagnosis ${ }^{\mathrm{d}}$} \\
\hline Low & $128(45)$ & $145(50)$ & $79(42)$ & $20(40)$ & 0.10 \\
\hline Intermediate & $118(41)$ & $90(31)$ & $69(36)$ & $20(40)$ & \\
\hline High & $41(14)$ & $53(18)$ & $42(22)$ & $10(20)$ & \\
\hline \multicolumn{6}{|l|}{ CAPRA score } \\
\hline Mean \pm SD & $2.6 \pm 1.6$ & $2.4 \pm 1.6$ & $2.8 \pm 1.7$ & $2.8 \pm 2.0$ & 0.11 \\
\hline Median & 2.0 & 2.0 & 2.0 & 2.5 & \\
\hline \multicolumn{6}{|l|}{ Treatment } \\
\hline $\mathrm{RP}$ & $198(68)$ & $189(65)$ & $99(51)$ & $23(45)$ & 0.002 \\
\hline Brachy \pm EBRT & $60(20)$ & $68(23)$ & $66(34)$ & $19(37)$ & \\
\hline EBRT & $35(12)$ & $36(12)$ & $28(15)$ & $9(18)$ & \\
\hline \multicolumn{6}{|l|}{ BMI $\left(\mathrm{kg} / \mathrm{m}^{2}\right)^{\mathrm{e}}$} \\
\hline$<25.0$ & $79(28)$ & $68(24)$ & $36(19)$ & $7(15)$ & 0.006 \\
\hline $25.0-29.9$ & $160(57)$ & $168(58)$ & $101(55)$ & $24(50)$ & \\
\hline$\geq 30$ & $44(16)$ & $53(18)$ & $48(26)$ & $17(35)$ & \\
\hline
\end{tabular}


Table 1 continued

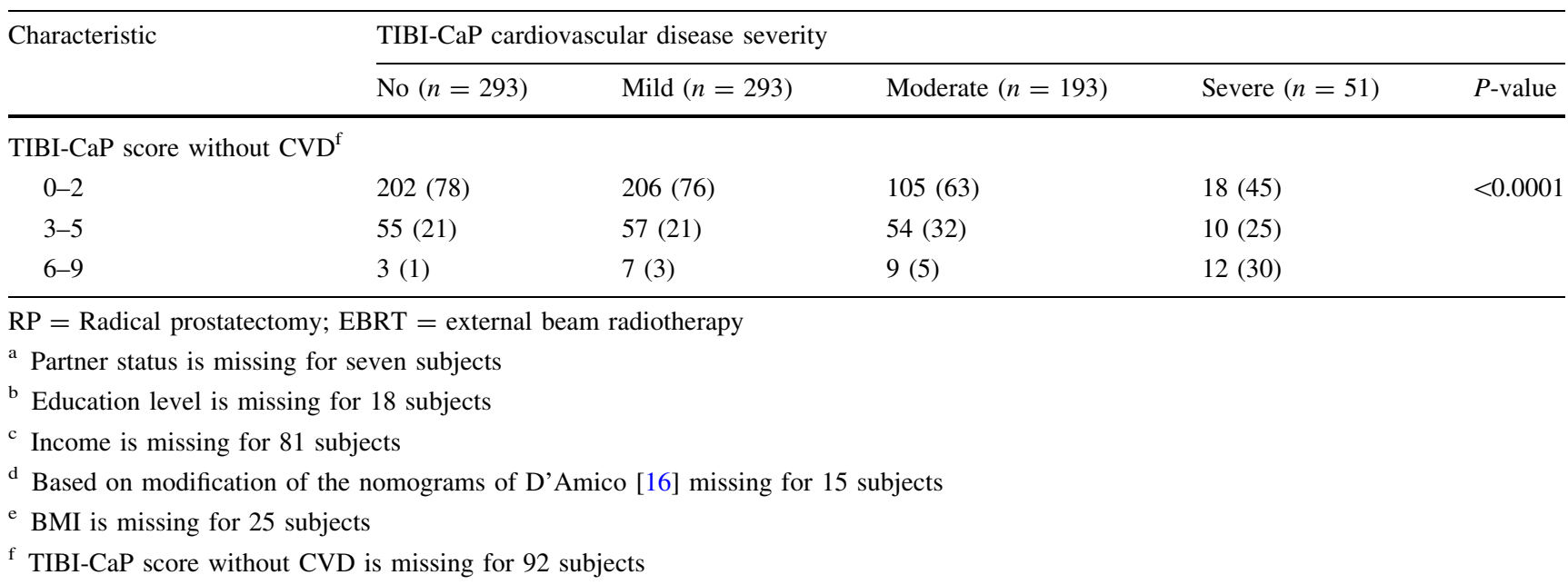

\section{Results}

Of the 830 men who met the inclusion criteria, $293(35 \%)$ reported no CVD, while 293 (35\%) were categorized with mild CVD, 193 (23\%) with moderate CVD, and 51 (6\%) with severe CVD. Men with increasing severity of CVD were older, more likely to be treated with brachytherapy or external beam radiation therapy, more likely to be overweight, and more likely to report comorbidities other than
CVD. Men with moderate or severe CVD were more likely to have less education and lower household income (Table 1).

Baseline generic and disease-specific HRQL was negatively associated with increasing CVD severity (raw means are presented in Table 2). Men with mild CVD had only slightly lower HRQL scores that were clinically and statistically similar to men without CVD. Men with moderate CVD scored worse on the physical, vitality, and general

Table 2 Health-related quality of life (raw means) for prostate cancer patients before treatment according to their TIBI-CaP cardiovascular disease severity

\begin{tabular}{|c|c|c|c|c|c|}
\hline \multirow{2}{*}{$\begin{array}{l}\text { Variable } \\
\text { SF-36 generic HRQL }\end{array}$} & \multicolumn{5}{|c|}{ TIBI-CaP cardiovascular disease severity } \\
\hline & $\begin{array}{l}\text { No }(n=293) \\
\text { Means } \pm \text { SD }\end{array}$ & $\begin{array}{l}\text { Mild }(n=293) \\
\text { Means } \pm \text { SD }\end{array}$ & $\begin{array}{l}\text { Moderate }(n=193) \\
\text { Means } \pm \text { SD }\end{array}$ & $\begin{array}{l}\text { Severe }(n=51) \\
\text { Means } \pm \mathrm{SD}\end{array}$ & $P$-value ${ }^{\mathrm{a}}$ \\
\hline Physical function & $93 \pm 13$ & $91 \pm 14$ & $79 \pm 21 * *$ & $68 \pm 27 * *$ & $<0.0001$ \\
\hline Role physical & $91 \pm 24$ & $88 \pm 27$ & $66 \pm 40^{* *}$ & $57 \pm 42 * *$ & $<0.0001$ \\
\hline Role emotional & $89 \pm 27$ & $89 \pm 26$ & $80 \pm 34^{*}$ & $67 \pm 39 * *$ & $<0.0001$ \\
\hline Vitality & $75 \pm 16$ & $72 \pm 16$ & $61 \pm 19^{* *}$ & $52 \pm 20^{* *}$ & $<0.0001$ \\
\hline Mental health & $81 \pm 15$ & $82 \pm 14$ & $77 \pm 16^{*}$ & $73 \pm 16^{*}$ & 0.02 \\
\hline Social function & $92 \pm 16$ & $92 \pm 16$ & $84 \pm 22 * *$ & $80 \pm 24^{*}$ & 0.0001 \\
\hline Bodily pain & $90 \pm 15$ & $88 \pm 18$ & $78 \pm 23^{* *}$ & $71 \pm 25^{* *}$ & $<0.0001$ \\
\hline General health & $80 \pm 16$ & $78 \pm 16$ & $65 \pm 20 * *$ & $55 \pm 23^{* *}$ & $<0.0001$ \\
\hline Mental component score & $53 \pm 8$ & $54 \pm 7$ & $51 \pm 10^{*}$ & $49 \pm 10^{*}$ & 0.004 \\
\hline Physical component score & $54 \pm 6$ & $53 \pm 7$ & $47 \pm 10^{* *}$ & $43 \pm 12 * *$ & $<0.0001$ \\
\hline \multicolumn{6}{|l|}{ UCLA prostate cancer index } \\
\hline Urinary function & $95 \pm 11$ & $93 \pm 11$ & $91 \pm 14^{* *}$ & $86 \pm 14^{*}$ & 0.009 \\
\hline Sexual function & $60 \pm 28$ & $54 \pm 30$ & $46 \pm 31$ & $33 \pm 30 * *$ & 0.005 \\
\hline Bowel function & $90 \pm 13$ & $91 \pm 11$ & $85 \pm 15^{* *}$ & $80 \pm 18^{*}$ & 0.0001 \\
\hline
\end{tabular}

${ }^{a} P$-values calculated for the independent effect of CVD severity, adjusted for age, race, educational level, partner status, income, BMI, treatment, and number of comorbidities other than CVD

*HRQL score statistically significantly different from men without CVD at the $P<0.05$ level

**HRQL score statistically significantly different from men without CVD at the $P<0.001$ level 
health domains of the SF-36. Men with severe CVD had worse generic and disease-specific HRQL than those without CVD.

Separate baseline analyses for men treated with RP or RT both revealed similar patterns of raw HRQL scores that worsened with increasing CVD severity, although some HRQL scale scores were lower for men treated with RT (Table 3). Direct comparison between both treatment groups showed statistically significant worse raw scores for the physical component summary score and sexual functioning among men treated with RT for all subgroups of CVD severity. However, these differences disappeared after adjustment for confounding variables (data not shown). Among men with severe CVD (TIBI-CVD score 4-7), those treated with RP had a mean TIBI-CVD score of $4.1(\mathrm{SD}=0.5)$, while those treated with $\mathrm{RT}$ had a mean

Table 3 Health-related quality of life for prostate cancer patients before treatment and changes within 6-month follow-up according to their TIBI-CaP cardiovascular disease severity

\begin{tabular}{|c|c|c|c|c|c|}
\hline \multirow[t]{2}{*}{ Radical prostatectomy } & \multicolumn{5}{|c|}{ TIBI-CaP cardiovascular disease severity } \\
\hline & $\begin{array}{l}\text { No }(n=159) \\
\text { Means } \pm \text { SD }\end{array}$ & $\begin{array}{l}\text { Mild }(n=159) \\
\text { Means } \pm \text { SD }\end{array}$ & $\begin{array}{l}\text { Moderate }(n=84) \\
\text { Means } \pm \text { SD }\end{array}$ & $\begin{array}{l}\text { Severe }(n=22) \\
\text { Means } \pm \mathrm{SD}\end{array}$ & $P$-value ${ }^{\mathrm{a}, \mathrm{b}}$ \\
\hline \multicolumn{6}{|l|}{ Mental component score } \\
\hline Before treatment & $53 \pm 9$ & $54 \pm 8$ & $51 \pm 10$ & $50 \pm 11$ & 0.04 \\
\hline Change within 6 months & $1 \pm 9$ & $0 \pm 7$ & $0 \pm 8$ & $-1 \pm 7$ & 0.55 \\
\hline \multicolumn{6}{|l|}{ Physical component score } \\
\hline Before treatment & $55 \pm 6$ & $54 \pm 6$ & $48 \pm 10$ & $48 \pm 10$ & $<0.0001$ \\
\hline Change within 6 months & $-4 \pm 9$ & $-3 \pm 8$ & $-1 \pm 9$ & $-1 \pm 8$ & 0.67 \\
\hline \multicolumn{6}{|l|}{ Urinary function } \\
\hline Before treatment & $95 \pm 11$ & $94 \pm 11$ & $91 \pm 14$ & $86 \pm 13$ & 0.08 \\
\hline Change within 6 months & $-31 \pm 27$ & $-31 \pm 28$ & $-27 \pm 28$ & $-25 \pm 29$ & 0.31 \\
\hline \multicolumn{6}{|l|}{ Sexual function } \\
\hline Before treatment & $64 \pm 26$ & $60 \pm 28$ & $53 \pm 29$ & $47 \pm 30$ & 0.10 \\
\hline Change within 6 months & $-40 \pm 27$ & $-41 \pm 25$ & $-37 \pm 27$ & $-34 \pm 24$ & 0.39 \\
\hline \multicolumn{6}{|l|}{ Bowel function } \\
\hline Before treatment & $90 \pm 14$ & $92 \pm 10$ & $85 \pm 14$ & $81 \pm 15$ & 0.0001 \\
\hline Change within 6 months & $-1 \pm 14$ & $-4 \pm 13$ & $-1 \pm 17$ & $-3 \pm 19$ & 0.28 \\
\hline Radiotherapy $^{\mathrm{c}}$ & No $(n=78)$ & Mild $(n=94)$ & Moderate $(n=85)$ & Severe $(n=22)$ & \\
\hline \multicolumn{6}{|l|}{ Mental component score } \\
\hline Before treatment & $54 \pm 7$ & $54 \pm 8$ & $52 \pm 10$ & $48 \pm 10$ & 0.24 \\
\hline Change within 6 months & $0 \pm 6$ & $0 \pm 7$ & $-2 \pm 10$ & $3 \pm 8$ & 0.58 \\
\hline \multicolumn{6}{|l|}{ Physical component score } \\
\hline Before treatment & $53 \pm 6$ & $51 \pm 9$ & $45 \pm 10$ & $39 \pm 11$ & $<0.0001$ \\
\hline Change within 6 months & $-3 \pm 7$ & $-2 \pm 7$ & $-2 \pm 9$ & $-3 \pm 8$ & 0.29 \\
\hline \multicolumn{6}{|l|}{ Urinary function } \\
\hline Before treatment & $95 \pm 11$ & $92 \pm 11$ & $90 \pm 14$ & $85 \pm 15$ & 0.09 \\
\hline Change within 6 months & $-11 \pm 17$ & $-10 \pm 15$ & $-11 \pm 19$ & $-10 \pm 13$ & 0.86 \\
\hline \multicolumn{6}{|l|}{ Sexual function } \\
\hline Before treatment & $50 \pm 30$ & $42 \pm 31$ & $39 \pm 30$ & $22 \pm 24$ & 0.18 \\
\hline Change within 6 months & $-18 \pm 24$ & $-18 \pm 26$ & $-14 \pm 23$ & $-3 \pm 17$ & 0.88 \\
\hline \multicolumn{6}{|l|}{ Bowel function } \\
\hline Before treatment & $91 \pm 11$ & $89 \pm 12$ & $84 \pm 15$ & $79 \pm 20$ & 0.07 \\
\hline Change within 6 months & $-7 \pm 22$ & $-7 \pm 16$ & $-9 \pm 20$ & $-13 \pm 17$ & 0.09 \\
\hline
\end{tabular}

${ }^{a} P$-values calculated for the independent effect of CVD severity on HRQL before treatment were adjusted for age, race, educational level, partner status, income, BMI, and number of comorbidities other than CVD

b $P$-values calculated for the independent effect of CVD severity on HRQL change within 6 months were adjusted for HRQL score before treatment, age, race, educational level, partner status, income, BMI, and number of comorbidities other than CVD

${ }^{c}$ Radiotherapy: Brachytherapy or external beam radiotherapy 
Fig. 1 Longitudinal changes in the generic health-related quality of life domain scores over time according to TIBICVD severity for men receiving radical prostatectomy (a) or radiotherapy (b)
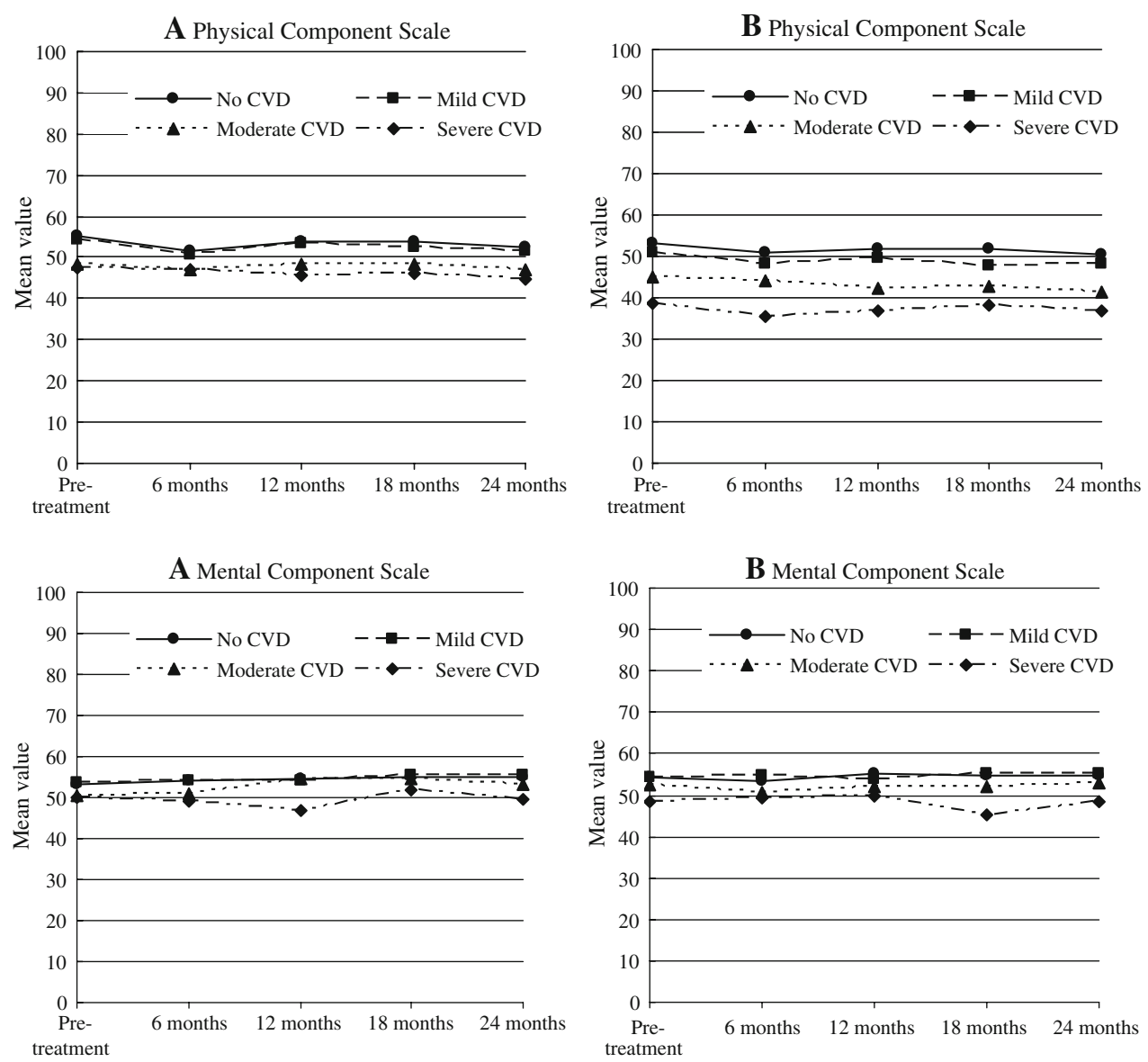

TIBI-CVD score of 4.6 ( $\mathrm{SD}=0.8)$, suggesting that within the severe CVD subgroup, men treated with RT had even more severe CVD.

The change (worsening) of generic and disease-specific HRQL in the first 6 months after treatment was independent of CVD severity (Table 3; Figs. 1 and 2). Multivariate analyses, controlling for the confounding effect of age, race, educational level, partner status, income, BMI, and comorbidities other than CVD, revealed no statistically significant effect of CVD on change in HRQL in the first 6 months.

During follow-up from 6 months up to 24 months after diagnosis, moderate or severe CVD was associated with worse physical (PCS) and mental (MCS) component summary scores and bowel function scores after adjustment for the confounding effect of baseline HRQL, age, race, education, partner status, income, BMI, treatment, and TIBI-CaP score without CVD (Table 4; Figs. 1 and 2). The differences in HRQL across CVD severity groups were parallel over time for the MCS and urinary and bowel function. However, for PCS and sexual function, we observed a significant negative interaction between CVD severity and time, suggesting that recovery after treatment was slower with increasing CVD severity. The interaction between CVD severity and treatment was not significant. HRQL trends over time for PCS, MCS, urinary, sexual and bowel function are provided in Figs. 1 and 2.

\section{Discussion}

This population-based analysis of 830 men treated with radical prostatectomy or radiation therapy for prostate cancer showed that men with moderate or severe cardiovascular disease had worse pre-treatment generic and disease-specific HRQL than did men without CVD; scores were even worse in men referred for RT. During 24 months of follow-up, men with moderate or severe CVD had worse absolute HRQL scores for the physical and mental summary scores of the SF-36 and for bowel function at all time points. Men with severe CVD also experienced a slower recovery in physical and sexual functioning than did men without CVD. CVD appears to have the same detrimental effect on recovery after surgery or radiation.

Treatment decisions made by prostate cancer patients may be influenced by non-clinical factors, such as age, comorbid diseases, or personal preferences. Our finding that men with moderate or severe CVD were more likely to 
Fig. 2 Longitudinal changes in disease-specific health-related quality of life domain scores over time according to TIBICVD severity for men receiving radical prostatectomy (a) or radiotherapy (b)
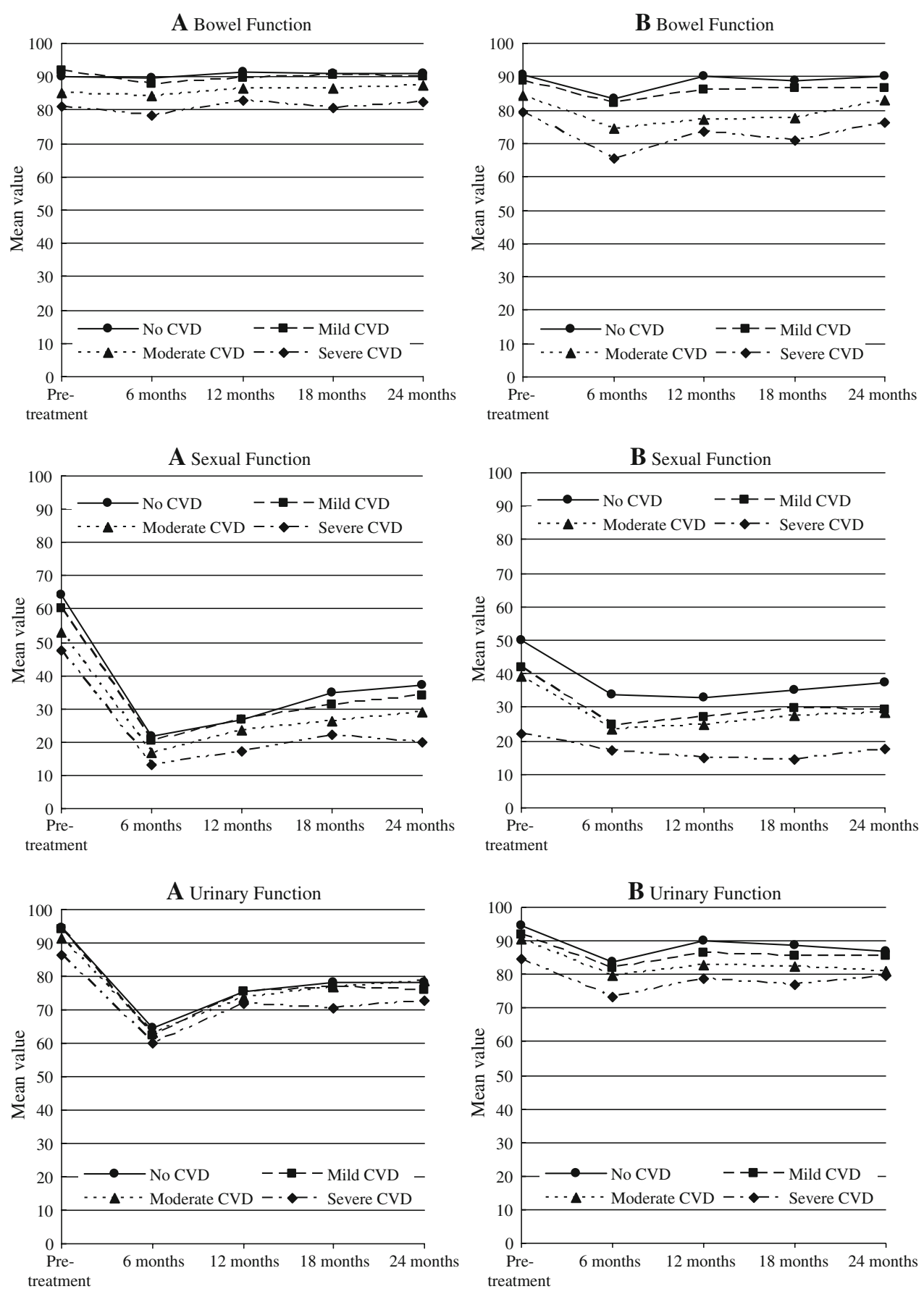

be treated with radiotherapy has been reported previously in the CaPSURE database [23] and is consistent with other studies showing that comorbidity was associated with lessaggressive treatment [3-6, 23]. We also found that within the strata of CVD severity, men treated with radiation tended to report worse HRQL at baseline than men who had surgery, suggesting that not only comorbidity severity, but also health status informs the consideration of treatment options. It remains unclear, however, whether comorbidity is also considered when anticipating long-term HRQL outcomes for a patient. And although comorbidity has often been incompletely described in HRQL outcome studies [12], the importance of its role is recognized in the delivery of quality care to elderly cancer patients [2, 24].

Self-reported comorbidity data has been shown to correlate highly with medical records abstraction and to be reproducible [25]. More specifically, results of the Prostate Cancer Outcomes Study (PCOS) showed that men with a relatively recent prostate cancer diagnosis are generally able to provide highly reliable reports of their concomitant health conditions [26]. Noteworthy was the positive correlation between severity of comorbidity and reliability in 
Table 4 Repeated measures analyses with mixed modeling evaluating the association between TIBI-CaP cardiovascular disease severity and HRQL during 24 months after diagnosis, adjusted for confounding variables

\begin{tabular}{|c|c|c|c|c|c|}
\hline \multirow[t]{2}{*}{ Variable } & \multicolumn{2}{|l|}{ SF 36} & \multicolumn{3}{|c|}{ UCLA prostate cancer index } \\
\hline & $\begin{array}{l}\text { PCS estimate } \\
\text { (SE) }\end{array}$ & $\begin{array}{l}\text { MCS estimate } \\
\text { (SE) }\end{array}$ & $\begin{array}{l}\text { Urinary function } \\
\text { estimate (SE) }\end{array}$ & $\begin{array}{l}\text { Sexual function } \\
\text { estimate (SE) }\end{array}$ & $\begin{array}{l}\text { Bowel function } \\
\text { estimate (SE) }\end{array}$ \\
\hline \multicolumn{6}{|l|}{ TIBI-CaP CVD severity ${ }^{\mathrm{a}}$} \\
\hline No & Reference & Reference & Reference & Reference & Reference \\
\hline Mild & $-0.6(0.4)$ & $-0.2(0.7)$ & $-1.1(1.6)$ & $-1.5(1.6)$ & $-1.8(0.8)$ \\
\hline Moderate & $-1.7(0.5)^{*}$ & $-1.3(0.6)^{*}$ & $-0.5(1.9)$ & $-0.6(1.8)$ & $-3.8(1.0)^{* *}$ \\
\hline Severe & $-3.2(0.8)^{* *}$ & $-3.7(0.9)^{* *}$ & $-0.0(3.1)$ & $-2.2(3.0)$ & $-5.2(1.6)^{*}$ \\
\hline Time* $^{*}$ TIBI-CaP CVD severity (interaction) ${ }^{\mathrm{a}}$ & 0.03 & NS & NS & 0.02 & NS \\
\hline Treatment* TIBI-CaP CVD severity (interaction) ${ }^{\mathrm{a}}$ & NS & NS & NS & NS & NS \\
\hline
\end{tabular}

${ }^{a} P$-values calculated for the independent effect of CVD severity, adjusted for baseline HRQL, age, race, educational level, partner status, income, BMI, treatment, number of comorbidities other than CVD

*HRQL score statistically significantly different from men without CVD at the $P<0.05$ level

**HRQL score statistically significantly different from men without CVD at the $P<0.001$ level

its reporting, which led PCOS investigators to conclude that condition severity should be obtained to increase the likelihood of obtaining reliable data. The recently validated TIBI-CaP was developed to provide a clinically useful and systematic method to assess non-cancer morbidity in men with prostate cancer [14]. In contrast to most other comorbidity measures, the TIBI-CaP includes the severity of conditions and was uniquely developed for use with prostate cancer as the index condition, as recommended by the NIH working group [2]. The aggregation of severity across conditions mitigates the potential for patients to report just one condition [14]-incorporating severity in comorbidity assessment improves validity of the data [26].

Our findings suggest that it is not simply the presence or absence of CVD, but its severity, that influences recovery after prostate cancer treatment: men with moderate to severe CVD reported significantly lower scores at baseline and follow-up. Still, even in this predominantly non-minority, affluent cohort, $29 \%$ of men had moderate to severe CVD. The prevalence of moderate to severe CVD is likely even higher in other prostate cancer populations that include more minority men with lower socioeconomic status. Although men with moderate CVD severity reported worse HRQL, only those with severe CVD reported a significantly slower recovery in physical and sexual functioning.

Although we were able to evaluate the effect of CVD on HRQL recovery in prostate cancer patients treated with RP and RT, the most frequently used treatments for men with localized prostate cancer [27], we did not include men treated with androgen deprivation therapy. The use of androgen deprivation with radiotherapy has become common, even in low-risk patients [28], but the interplay between CVD and androgen deprivation deserves further attention. A recent analysis of 1,372 men from three randomized trials showed that the use of androgen deprivation was associated with fatal myocardial infarction in men 65 years or older [29]. Although the mechanisms responsible for this effect are unclear, the authors recommend that men of advanced age considering RT and androgen deprivation for prostate cancer should be undergo cardiovascular evaluation before initiation of therapy [29].

Our study has several limitations. Because subjects completed the TIBI-CaP within 24 months of diagnosis, the presence and/or severity of comorbidity may have changed since baseline. However, because baseline HRQL scores corresponded well with the increasing level of CVD severity and differed in the expected direction by treatment group, comorbidity misclassification bias seems unlikely. In addition, CaPSURE is an observational database that has been designed to track longitudinal health outcomes for patients with prostate cancer. Clinical sites have been selected to represent a breadth of practice settings (managed care, fee-for-service practices, university teaching hospitals) [5]. Although geographic representation is spread throughout all regions of the US, the sample is not as ethnically diverse as the overall population of US men with prostate cancer [11]. The fact that we observed a low mean TIBI-CVD score in our sample suggests that we evaluated a relatively healthy population of prostate cancer patients. It is therefore possible that we underestimated the prevalence of prostate cancer patients with moderate to severe CVD.

One strength of this study is the use of baseline HRQL, known to be a strong predictor of future outcomes [21, 22]. Furthermore, the analysis of HRQL data from four time points after diagnosis was optimized with mixed modeling, giving us an ability to observe changes in HRQL over the course of disease.

In conclusion, the observations of our longitudinal analyses suggest that prostate cancer patients with moderate to severe CVD have worse HRQL during follow-up and 
that men with severe CVD recover their physical and sexual functioning more slowly after treatment. Although men treated with radiation tended to have worse baseline scores than men treated with surgery, CVD severity had the same detrimental effect on HRQL during follow-up in both groups. Because the prognosis in prostate cancer is generally good, men may wish to consider the expected impact of comorbidity on long-term HRQL outcomes when deciding on primary treatment. Future studies should also examine the impact of long-term androgen ablation on cardiovascular outcomes.

Open Access This article is distributed under the terms of the Creative Commons Attribution Noncommercial License which permits any noncommercial use, distribution, and reproduction in any medium, provided the original author(s) and source are credited.

\section{References}

1. SEER Cancer Statistics Review, 1975-2004, In L. A. G Ries, D. Melbert, M. Krapcho, et al. (Eds.). Bethesda, MD, National Cancer Institute, 2007, pp based on November 2006 SEER data submission, posted to the SEER web site, 2007.

2. Yancik, R., Ganz, P. A., Varricchiom, C. G., et al. (2001). Perspectives on comorbidity and cancer in older patients: Approaches to expand the knowledge base. Journal of Clinical Oncology, 19, 1147-1151.

3. Hall H. I., Satariano W. A., Thompson, T., et al. (2002). Initial treatment for prostate carcinoma in relation to comorbidity and symptoms. Cancer, 95, 2308-2315. doi:10.1002/cncr.10926.

4. Harlan, L. C., Potosky, A., Gilliland, F. D., et al. (2001). Factors associated with initial therapy for clinically localized prostate cancer: Prostate cancer outcomes study. Journal of the National Cancer Institute, 93, 1864-1871. doi:10.1093/jnci/93.24.1864.

5. Houterman, S., Janssen-Heijnen, M. L., Hendrikx, A. J., et al. (2006). Impact of comorbidity on treatment and prognosis of prostate cancer patients: A population-based study. Critical Reviews in Oncology/Hematology, 58, 60-67. doi:10.1016/j. critrevonc.2005.08.003.

6. van de Poll-Franse, L. V., Houterman, S., Janssen-Heijnen, M. L., et al. (2007). Less aggressive treatment and worse overall survival in cancer patients with diabetes: A large population based analysis. International Journal of Cancer, 120, 1986-1992. doi: 10.1002/ijc.22532.

7. Alibhai, S. M., Leach, M., Tomlinson, G., et al. (2005). 30-day mortality and major complications after radical prostatectomy: Influence of age and comorbidity. Journal of the National Cancer Institute, 97, 1525-1532.

8. Surveillance Epidemiology, and End Results (SEER) Program (www.seer.cancer.gov) SEER*Stat Database: Incidence-SEER 17 Regs Limited-Use, Nov 2006 Sub (1973-2004 varying), National Cancer Institute, DCCPS, Surveillance Research Program, Cancer Statistics Branch, released April 2007, based on the November 2006 submission.

9. Arredondo, S. A., Elkin, E. P., Marr, P. L., et al. (2006). Impact of comorbidity on health-related quality of life in men undergoing radical prostatectomy: Data from CaPSURE. Urology, 67, 559-565. doi:10.1016/j.urology.2005.09.006.

10. Hu, J. C., Elkin E. P., Pasta, D. J., et al. (2004). Predicting quality of life after radical prostatectomy: Results from CaPSURE. The Journal of Urology, 171, 703-707. discussion 707-8.
11. Latini, D. M., Chan, J. M., Cowan, J. E., et al. (2006). Healthrelated quality of life for men with prostate cancer and diabetes: A longitudinal analysis from CaPSURE. Urology, 68, 1242-1247. doi:10.1016/j.urology.2006.08.1096.

12. Ramsey, S. D., Zeliadt, S. B., Hall, I. J., et al. (2007). On the importance of race, socioeconomic status and comorbidity when evaluating quality of life in men with prostate cancer. The Journal of Urology, 177, 1992-1999. doi:10.1016/j.juro.2007. 01.138 .

13. Herold, D. M., Hanlon, A. L., \& Hanks, G. E. (1999). Diabetes mellitus: A predictor for late radiation morbidity. International Journal of Radiation Oncology, Biology, Physics, 43, 475-479. doi:10.1016/S0360-3016(98)00460-X.

14. Litwin, M. S., Greenfield, S., Elkin, E. P., et al. (2007). Assessment of prognosis with the total illness burden index for prostate cancer: Aiding clinicians in treatment choice. Cancer, 109, 17771783. doi:10.1002/cncr.22615.

15. Lubeck, D. P., Litwin, M. S., Henning, J. M., et al. (1996). The CaPSURE database: A methodology for clinical practice and research in prostate cancer. CaPSURE Research Panel. Cancer of the Prostate Strategic Urologic Research Endeavor. Urology, 48, 773-777. doi:10.1016/S0090-4295(96)00226-9.

16. D'Amico, A. V., Whittington, R., Malkowicz, S. B., et al. (1998). Biochemical outcome after radical prostatectomy, external beam radiation therapy, or interstitial radiation therapy for clinically localized prostate cancer. JAMA, 280, 969-974. doi:10.1001/ jama.280.11.969.

17. Greenfield, S., Sullivan, L., Dukes, K. A., et al. (1995). Development and testing of a new measure of case mix for use in office practice. Medical Care, 33, AS47-AS55.

18. Stier, D. M., Greenfield, S., Lubeck, D. P., et al. (1999). Quantifying comorbidity in a disease-specific cohort: Adaptation of the total illness burden index to prostate cancer. Urology, 54, 424429. doi:10.1016/S0090-4295(99)00203-4.

19. Hays, R. D., Sherbourne, C. D., \& Mazel, R. M. (1993). The RAND 36-Item Health Survey 1.0. Health Economics, 2, $217-$ 227. doi: $10.1002 /$ hec. 4730020305 .

20. Litwin, M. S., Hays, R. D., Fink, A., et al. (1998). The UCLA Prostate Cancer Index: Development, reliability, and validity of a health-related quality of life measure. Medical Care, 36, 10021012. doi:10.1097/00005650-199807000-00007.

21. Talcott, J. A., Manola, J., Clark J. A., et al. (2003). Time course and predictors of symptoms after primary prostate cancer therapy. Journal of Clinical Oncology, 21, 3979-3986. doi:10.1200/JCO. 2003.01.199.

22. Stanford, J. L., Feng, Z., Hamilton, A. S., et al. (2000). Urinary and sexual function after radical prostatectomy for clinically localized prostate cancer: The Prostate Cancer Outcomes Study. JAMA, 283, 354-360. doi:10.1001/jama.283.3.354.

23. Litwin, M. S., Flanders, S. C., Pasta, D. J., et al. (1999). Sexual function and bother after radical prostatectomy or radiation for prostate cancer: Multivariate quality-of-life analysis from $\mathrm{CaP}$ SURE. Cancer of the Prostate Strategic Urologic Research Endeavor. Urology, 54, 503-508. doi:10.1016/S0090-4295(99) 00172-7.

24. Rowland, J. H., \& Yancik, R. (2006). Cancer survivorship: The interface of aging, comorbidity, and quality care. Journal of the National Cancer Institute, 98, 504-505.

25. Katz, J. N., Chang, L. C., Sangha, O., et al. (1996). Can comorbidity be measured by questionnaire rather than medical record review? Medical Care, 34, 73-84. doi:10.1097/00005650199601000-00006.

26. Klabunde, C. N., Reeve, B. B., Harlan, L. C., et al. (2005). Do patients consistently report comorbid conditions over time?: Results from the prostate cancer outcomes study. Medical Care, 43, 391-400. doi:10.1097/01.mlr.0000156851.80900.d1. 
27. Penson D. F., \& Chan, J. M. (2007). Prostate Cancer. In M. S. Litwin, C. S. Saigal (Eds.), Urologic diseases in America (pp. 71122). Washington, DC: US Government Publishing Office. NIH Publication No. 07-5512, US Department of Health and Human Services, Public Health Service, National Institutes of Health, National Institute of Diabetes and Digestive and Kidney Diseases.

28. Zeliadt, S. B., Potosky, A. L., Penson, D. F., et al. (2006). Survival benefit associated with adjuvant androgen deprivation therapy combined with radiotherapy for high- and low-risk patients with nonmetastatic prostate cancer. International Journal of Radiation Oncology, Biology, Physics, 66, 395-402. doi: 10.1016/j.ijrobp.2006.04.048.

29. D'Amico, A. V., Denham, J. W., Crook. J., et al. (2007). Influence of androgen suppression therapy for prostate cancer on the frequency and timing of fatal myocardial infarctions. Journal of Clinical Oncology, 25, 2420-2425. doi:10.1200/JCO.2006.09.3369. 